\title{
The 'Core' of Symmetric Homogeneous Polynomial Inequalities of Degree Four of Three Real Variables
}

\author{
Mariyan Milev ${ }^{1}$, Nedelcho Milev ${ }^{2}$ \\ ${ }^{1}$ Department of Mathematics and Physics, UFT-Plovdiv, bul. Maritza 26, 4000 Plovdiv, \\ Bulgaria, tel. +359 32603744 email: marianmilev2002@gmail.com \\ 2 Department of Mathematical Analysis, Plovdiv University 'Paisii Hilendarski', Tzar \\ Asen 24, 4000 Plovdiv, Bulgaria, phone: +359-32 519542, email: milev0@abv.bg
}

\begin{abstract}
In this paper we explore inequalities between symmetric homogeneous polynomials of degree four of three real variables and three nonnegative real variables. The main theorems describe the cases in which the smallest possible coefficient is not expressed by the other coefficients. The problem is resolved by introducing a parametric representation.
\end{abstract}

Key words: symmetric, homogeneous, polynomial, inequalities, degree three and four.

Mathematics Subject Classification: 26 D05.

\section{Introduction}

In recent years, inequalities between symmetric homogeneous polynomials are studied in several articles [1]-[10]. We will explore the inequality

$$
\begin{gathered}
x^{4}+y^{4}+z^{4}+a\left(x y^{3}+y z^{3}+z x^{3}+x^{3} y+y^{3} z+z^{3} x\right)+b\left(x^{2} y^{2}+y^{2} z^{2}+z^{2} x^{2}\right)+ \\
+c x y z(x+y+z) \geq 0,
\end{gathered}
$$

where $a, b, c$ are real constants, and $x, y, z$ are real variables or nonnegative real variables. The proof of the main theorem 3 in this paper is structured by setting of auxiliary functions (2), lemma 4 and the representation (7). For brevity we will denote the main symmetric homogeneous polynomials of degree four

$$
w_{4}=x^{4}+y^{4}+z^{4}, \quad w_{3}=x^{3} y+y^{3} z+z^{3} x+x y^{3}+y z^{3}+z x^{3}, \quad w_{2}=x^{2} y^{2}+y^{2} z^{2}+z^{2} x^{2}
$$

$w_{1}=x y z(x+y+z)$ and the inequality is written in the form

$$
f(x, y, z)=w_{4}+a w_{3}+b w_{2}+c w_{1} \geq 0 .
$$

In Section I we find $b_{\text {min }}$ such that the inequality $f(x, y, z)=w_{4}+a w_{3}+b w_{2}+c w_{1} \geq 0$ holds for arbitrary $x, y, z \in R$ if and only if when $b \geq b_{\text {min }}$.

In the particular case $a=-\frac{1}{2}$ the upper inequality holds if and only if when $b_{\text {min }}=\max \left(-c ; \frac{c}{2}-\frac{9}{8}\right)$ (Theorem 2$)$.

When $c \leq-a^{2}-2 a$ (Theorem 1) $b_{\text {min }}=-2 a-c-1$. This statement follows also from [4], Theorem 2.1. 
When $c>-a^{2}-2 a$ the situation radically changes. In some particular cases $b_{\text {min }}$ could be expressed in radicals, for example when $a=2$ and $c=4>-a^{2}-2 a$ we find

$b_{\text {min }}=\frac{(5 \sqrt{5}-7)}{2}$. When $b=b_{\text {min }}$ the equality holds when $x=y=1$ and $z=-\frac{1+\sqrt{5}}{2}$ and of course for any permutation. When $a=-1$ and $c=2>-a^{2}-2 a$ we find $b=\frac{2+5 \sqrt[3]{100}-10 \sqrt[3]{10}}{12}=0.305299773 \ldots$ The equality holds when $x=y=1$ and $z=\frac{\sqrt[3]{100}-2 \sqrt[3]{10}-2}{6}=-0.27788 \ldots$

We overcome this problem by introducing the rational parameterization (2) (see Theorem 3).

We note that this parametrization is in line with [5], Theorem 2.2: 'The inequality $f(x, y, z)=w_{4}+a w_{3}+b w_{2}+c w_{1} \geq 0$ holds if and only if when $f(x, 1,1) \geq 0$ for every real $x$.'

In Section II we will explore the inequality

$$
f(x, y, z)=w_{4}+a w_{3}+b w_{2}+c w_{1} \geq 0,
$$

where $x, y, z$ are nonnegative real variables.

First we find the necessarily conditions for the coefficient $b$ (Lemma 5) and then we search $c_{\text {min }}$ such that the previous inequality holds if and only if when $c \geq c_{\min }$. We note that $w_{1}$ is the smallest symmetric polynomial $2 w_{4} \geq w_{3} \geq 2 w_{2} \geq 2 w_{1} \geq 0$.

When $a \geq-1 \Rightarrow b \geq-2(a+1)$ and $c_{\min }=-2 a-b-1$. This statement follows also from [4], Theorem 2.6. According to this theorem the inequality $f(x, y, z) \geq 0$ holds also when $a<-1$, but when $c>-2 a-b-1$ the condition $b \geq a^{2}-1$ is not necessary. When $a<-1$ and $b<a^{2}-1$, only in some particular cases $c_{\text {min }}$ could be expressed in radicals. For example, when $a=-4$ and $b=14\left(-2(a+1)<b<a^{2}-1\right)$ $c_{\min }=4[-9+8 \sqrt{2}-2(3-\sqrt{2}) \sqrt{3-\sqrt{2}}]=-6.72076 \ldots$ When $c=c_{\min }$ the equality holds when $x=y=1$ and $z=\sqrt{2}+\sqrt{6-2 \sqrt{2}}=3.1951 \ldots$ and of course for any permutation. Another example, when $a=-6, b=31$

$c_{\text {min }}=-58+20 \sqrt{15}+(5-3 \sqrt{15}) \sqrt{40-2 \sqrt{15}}=-18.131094 \ldots\left(\frac{a^{2}}{4}+2<b<a^{2}-1\right)$.

The equality holds when $x=y=1$ and $z=\frac{1+\sqrt{15}+\sqrt{40-2 \sqrt{15}}}{2}=5.276 \ldots$ 7).

We again solve the problem by using the rational parametrization (2) (see Theorem

We note that this do not contradict to [2] and [6], Theorem 2.1: 'The inequality $f(x, y, z)=w_{4}+a w_{3}+b w_{2}+c w_{1} \geq 0$ holds if and only if when $f(x, 1,0) \geq 0$ and $f(x, 1,1) \geq 0$ for every $x \geq 0$.'

Remark. All identities are verified via the Maplesoft platform.

\section{Main Results}

Section I. We will explore the inequality

$$
f(x, y, z)=w_{4}+a w_{3}+b w_{2}+c w_{1} \geq 0,
$$

where $a, b, c$ are real constants and $x, y, z$ are real variables. 
Theorem 1. One necessary condition to be true the inequality (1) is $b \geq-2 a-c-1$. When $c \leq-a^{2}-2 a$ this condition is also a sufficient condition (This statement follows also from [4], Theorem 2.1).

Theorem 2. When $a=-\frac{1}{2}$ the inequality (1) holds if and only if when $b \geq \max \left(-c, \frac{c}{2}-\frac{9}{8}\right)$.

When $a \neq-\frac{1}{2}$ and $c \geq-a^{2}-2 a$ we introduce the rational functions:

$$
\begin{aligned}
& c(t)=\frac{2 t^{4}+2(a+1) t^{3}+(2 a+4) t^{2}+(3 a+4) t-a}{2 t+1}, \\
& b(t)=\frac{t^{4}+(a+4) t^{3}+(5 a+4) t^{2}+4(a+1) t+2 a+2}{-(2 t+1)}
\end{aligned}
$$

and we will explore the different cases about the parameter $a$ when the rational function $c(t)$ always takes values in the interval $\left[-a^{2}-2 a ;+\infty\right)$.

When $-\frac{1}{2}<a \leq 4$ and $t \in\left[-a-1 ;-\frac{1}{2}\right)$ the continuous function $c(t)$ is monotone increasing and $-a^{2}-2 a=c(-a-1) \leq c(t)<c\left(-\frac{1}{2}-0\right)=+\infty$.

When $-2 \leq a<-\frac{1}{2}$ and $t \in\left(-\frac{1}{2} ;-a-1\right]$ the continuous function $c(t)$ is monotone decreasing and $+\infty=c\left(-\frac{1}{2}+0\right)>c(t) \geq c(-a-1)=-a^{2}-2 a$.

Let us set

$$
t_{1}=\frac{-a-\sqrt{(a+2)(a-4)}}{2} \quad \text { and } \quad t_{2}=\frac{-a+\sqrt{(a+2)(a-4)}}{2}
$$

When $a>4$ we obtain two intervals $t \in\left[-a-1 ; t_{1}\right] \cup\left[t_{2} ;-\frac{1}{2}\right)$,

$-a-1<t_{1}<-2<t_{2}<-\frac{1}{2}$. The function $c(t)$ is monotone increasing and $-a^{2}-2 a=c(-a-1)<c\left(t_{1}\right)=c\left(t_{2}\right)=\frac{a^{2}}{2}+2<c\left(-\frac{1}{2}-0\right)=+\infty$.

When $a<-2$ we obtain again two intervals: $t \in\left(-\frac{1}{2} ; t_{1}\right] \cup\left[-a-1 ; t_{2}\right]$. The function $c(t)$ is monotone decreasing in $\left(-\frac{1}{2} ; t_{1}\right]$ and monotone increasing in $\left[-a-1 ; t_{2}\right]$ : $+\infty=c\left(-\frac{1}{2}+0\right)>c\left(t_{1}\right)=c\left(t_{2}\right)=\frac{a^{2}}{2}+2>c(-a-1)=-a^{2}-2 a$.

Theorem 3. When $a \neq-\frac{1}{2}$ and $c \geq-a^{2}-2 a$ in the following four cases:

(3.1.) $-\frac{1}{2}<a \leq 4$ and $t \in\left[-a-1 ;-\frac{1}{2}\right)$;

(3.2.) $\quad-2 \leq a<-\frac{1}{2}$ and $t \in\left(-\frac{1}{2} ;-a-1\right]$; 


$$
\begin{aligned}
& a>4 \text { and } t \in\left[-a-1 ; t_{1}\right] \cup\left[t_{2} ;-\frac{1}{2}\right) ; \\
& a<-2 \text { and } t \in\left(-\frac{1}{2} ; t_{1}\right] \cup\left[-a-1 ; t_{2}\right]
\end{aligned}
$$

the inequality $f(x, y, z)=w_{4}+a w_{3}+b w_{2}+c w_{1} \geq 0$ holds if and only if when $b \geq b(t)$. When $b=b(t)$ we have $f(t, 1,1)=f(1, t, 1)=f(1,1, t)=0$.

For the proof of this theorem 3 and theorem 7 below we will use the following known statement.

Lemma 4. For arbitrary real numbers $X, Y$ and $Z$ the inequality

$$
g(X, Y, Z)=X^{2}+Y^{2}+Z^{2}+k(X Y+Y Z+Z X) \geq 0
$$

holds if and only if when $-1 \leq k \leq 2$.

Section II. We will explore the inequality

$$
f(x, y, z)=w_{4}+a w_{3}+b w_{2}+c w_{1} \geq 0,
$$

where $a, b, c$ are real constants and $x, y, z$ are nonnegative real variables.

Lemma 5. To be true the inequality (5) it is necessary:

$b \geq\left\{\begin{array}{ll}\frac{1}{4} a^{2}+2 & \text { when } a \leq-4 ; \\ -2(a+1) & \text { when } a \geq-4\end{array}\right.$ and $c \geq-2 a-b-1$.

Theorem 6. ([4], Theorem 2.6). When $a \geq-1$ the inequality (5) holds if and only if when $b \geq-2(a+1)$ and $c \geq-2 a-b-1$.

Remark. According to ([4], Theorem 2.6) the inequality (5]) is true also when $a<-1$, $b \geq a^{2}-1$ and $c \geq-2 a-b-1$, but when $c>-2 a-b-1$ the condition $b \geq a^{2}-1$ is not necessary.

When $a<-1$ and $b \leq a^{2}-1$ we will describe the different cases about the parameter $a$ again with the rational function $b(t)$ and $c(t)$, defined by (2) .

When $-2 \leq a<-1$ and $t \in[0 ;-a-1]$ the rational function $b(t)$ is monotone increasing and takes values in the intervala $\left[-2(a+1) ; a^{2}-1\right]$, i.e.

$-2(a+1)=b(0) \leq b(t) \leq b(-a-1)=a^{2}-1$.

When $-4<a<-2$ there are two intervals for $t$.

When $t \in\left[0 ; t_{1}\right]$ the function $b(t)$ is monotone increasing and takes values in the interval $\left[-2(a+1) ; \frac{a^{2}}{4}+2\right]$, i.e. $-2(a+1)=b(0) \leq b(t) \leq b\left(t_{1}\right)=\frac{a^{2}}{4}+2$, and when $t \in\left[-a-1 ; t_{2}\right]$ the function $b(t)$ is monotone decreasing and takes values in the interval $\left[\frac{a^{2}}{4}+2 ; a^{2}-1\right]$, i.e. $\frac{a^{2}}{4}+2=b\left(t_{2}\right) \leq b(t) \leq b(-a-1)=a^{2}-1$, where $t_{1}$ and $t_{2}$ are defined in formulas (3).

When $a \leq-4$ and $t \in\left[-a-1 ; t_{2}\right]$ the function $b(t)$ is monotone decreasing and takes values in the interval $\left[\frac{a^{2}}{4}+2 ; a^{2}-1\right]$, i.e. $\frac{a^{2}}{4}+2=b\left(t_{2}\right) \leq b(t) \leq b(-a-1)=a^{2}-1$. 
Theorem 7. In the three cases:

(7.1.) $\quad-2 \leq a<-1$ and $t \in[0 ;-a-1]$;

(7.2.) $-4<a<-2$ and $t \in\left[0 ; t_{1}\right] \cup\left[-a-1 ; t_{2}\right]$;

(7.3.) $\quad a \leq-4$ and $t \in\left[-a-1 ; t_{2}\right]$

the inequality $f(x, y, z)=w_{4}+a w_{3}+b(t) w_{2}+c w_{1} \geq 0$ holds if and only if when $c \geq c(t)$.

When $c=c(t)$ we have $f(t, 1,1)=f(1, t, 1)=f(1,1, t)=0$.

\section{Proves of Theorems}

Section I (real variables)

Remark. In the proves we have in mind that the following basic symmetric homogeneous polinomial inequalites of degree four of three real variables are true:

$2 w_{4} \geq w_{3}, \quad w_{4} \geq w_{2} \geq 0, \quad w_{2} \geq w_{1}$ and $w_{2}+2 w_{1} \geq 0$.

\section{Proof of Theorem 1.}

The necessity follows from $0 \leq f(1,1,1)=3+6 a+3 b+3 c$, i.e. $b \geq-2 a-c-1$.

The sufficiency follows from the relation

$$
w_{4}+a w_{3}+\left(a^{2}-1\right) w_{2}-\left(a^{2}+2 a\right) w_{1}=u^{2}+v^{2}-u v \geq 0,
$$

where $u=(x-y)(x+y+a z)$ and $v=(x-z)(x+z+a y)$.

When $c \leq-a^{2}-2 a \Rightarrow b \geq-2 a-c-1 \geq a^{2}-1$ and $w_{4}+a w_{3}+b w_{2}+c w_{1}=$

$$
=\left[w_{4}+a w_{3}+\left(a^{2}-1\right) w_{2}-\left(a^{2}+2 a\right) w_{1}\right]+(2 a+b+c+1) w_{2}-\left(c+a^{2}+2 a\right)\left(w_{2}-w_{1}\right) \geq 0 .
$$

Remark. When $a=-1, c=-a^{2}-2 a=1$ and $b=-2 a-c-1=0$ we obtain the famous inequality of I. Schur [11], $w_{4}+w_{1} \geq w_{3}$.

\section{Proof Theorem 2.}

When $a=-\frac{1}{2}$ and $c \leq-a^{2}-2 a=\frac{3}{4} \Rightarrow b \geq-2 a-c-1=-c \geq-\frac{3}{4}$ the basic inequality (6) is $w_{4}-\frac{1}{2} w_{3}-\frac{3}{4} w_{2}+\frac{3}{4} w_{1} \geq 0$ and the statement follows from Theorem 1 .

From $0 \leq f(2,2,-1)=24 b-12 c+27 \Rightarrow b \geq \frac{c}{2}-\frac{9}{8}$.

When $c \geq \frac{3}{4}$ and $b \geq \frac{c}{2}-\frac{9}{8}$ we have

$w_{4}-\frac{1}{2} w_{3}+b w_{2}+c w_{1}=\left(w_{4}-\frac{1}{2} w_{3}-\frac{3}{4} w_{2}+\frac{3}{4} w_{1}\right)+\frac{1}{2}\left(c-\frac{3}{4}\right)\left(w_{2}+2 w_{1}\right)+\left(b-\frac{c}{2}+\frac{9}{8}\right) w_{2} \geq 0$.

Before the proof of Theorem 3 we will prove Lemma 4.

\section{Proof of Lemma 4.}

From $g(1,1,1)=3+3 k \geq 0$ and from $g(1,-1,0)=2-k \geq 0$ it follows that $-1 \leq k \leq 2$. And $g(X, Y, Z)=\frac{k+1}{3}(X+Y+Z)^{2}+\frac{2-k}{3}\left(X^{2}+Y^{2}+Z^{2}-X Y-Y Z-Z X\right) \geq 0$. 
Proof of Theorem 3. Necessity.

$f(x, y, z)=w_{4}+a w_{3}+b w_{2}+c(t) w_{1}=w_{4}+a w_{3}+b(t) w_{2}+c(t) w_{1}+(b-b(t)) w_{2}$.

From $0 \leq f(t, 1,1)=0+(b-b(t))\left(2 t^{2}+1\right)$ follows that $b \geq b(t)$.

Sufficiency follows from the identity:

$$
\begin{gathered}
w_{4}+a w_{3}+b(t) w_{2}+c(t) w_{1}=X^{2}+Y^{2}+Z^{2}+k(X Y+Y Z+Z X), \text { where } \\
X=x^{2}+p x y+p x z+q y z, Y=y^{2}+p y z+p y x+q z x, Z=z^{2}+p z x+p z y+q x y, \\
p=-\frac{t^{2}+t+1}{2 t+1}, q=\frac{t^{2}+2 t}{2 t+1} \text { and } k=\frac{2 t^{2}+2 t(a+1)+a+2}{t-1}
\end{gathered}
$$

When $t=1$ we obtain that $p=-1, q=1$ and the indentity do not depend on $k$ because $X Y+Y Z+Z X=0$.

Having in mind Lemma 4 it remains to check that $-1 \leq k \leq 2$ for the different cases.

When $-\frac{1}{2}<a \leq 4$ and $t \in\left[-a-1 ;-\frac{1}{2}\right) \Rightarrow 2 t+1<0, t-1<0, t+a+1 \geq 0 \Rightarrow$

$$
k+1=\frac{(2 t+1)(t+a+1)}{t-1} \geq 0 \text { and } k-2=\frac{(2 t+a)^{2}+(4-a)(a+2)}{2(t-1)}<0 .
$$

(3.2) When $-2<a<-1$ and $t \in\left(-\frac{1}{2} ;-a-1\right] \Rightarrow 2 t+1>0, t-1 \leq-a-2<0$, $t+a+1<0 \Rightarrow$

$$
k+1=\frac{(2 t+1)(t+a+1)}{t-1} \geq 0 \text { and } k-2=\frac{(2 t+a)^{2}+(4-a)(a+2)}{2(t-1)}<0 .
$$

When $a=-2$ and $t \in\left(-\frac{1}{2} ;-a-1\right) \Rightarrow k=2 t \in(-1 ; 2)$.

Remark. The case $t=1$ is possible only if $a=-2 \Rightarrow b(1)=3, c(1)=0$ and

$$
2\left(w_{4}-2 w_{3}+3 w_{2}\right)=(y-z)^{4}+(x-z)^{4}+(x-y)^{4}=2\left(x^{2}+y^{2}+z^{2}-x y-y z-x z\right)^{2} .
$$

(3.3) When $a>4$ and $t \in\left[-a-1 ; t_{1}\right] \cup\left[t_{2} ;-\frac{1}{2}\right) \Rightarrow 2 t+1<0, t-1<0, t+a+1 \geq 0$

$$
\Rightarrow \quad k+1=\frac{(2 t+1)(t+a+1)}{t-1} \geq 0 \text { and } k-2=\frac{2\left(t-t_{1}\right)\left(t-t_{2}\right)}{t-1} \leq 0 .
$$

(3.4) Case: $a<-2$ and $t \in\left(-\frac{1}{2} ; t_{1}\right] \cup\left[-a-1 ; t_{2}\right]$.

When $t \in\left(-\frac{1}{2} ; t_{1}\right] \Rightarrow t \leq t_{1}<1, \quad 2 t+1>0, \quad t+a+1<0$, i.e.

$$
k+1=\frac{(2 t+1)(t+a+1)}{t-1} \geq 0 \text { and } k-2=\frac{2\left(t-t_{1}\right)\left(t-t_{2}\right)}{t-1} \leq 0 .
$$

When $t \in\left[-a-1 ; t_{2}\right] \Rightarrow t>1, \quad t+a+1 \geq 0$ and again

$$
k+1=\frac{(2 t+1)(t+a+1)}{t-1} \geq 0 \text { and } k-2=\frac{2\left(t-t_{1}\right)\left(t-t_{2}\right)}{t-1} \leq 0 .
$$


Remark. The function $b(t)$ is not monotone, e.g. when

$a=2, t=-1 \Rightarrow c(-1)=8, b(-1)=3, k=0, p=q=1$ and

$w_{4}+2 w_{3}+3 w_{2}+8 w_{1}=\left(x^{2}+x y+x z+y z\right)^{2}+\left(y^{2}+x y+x z+y z\right)^{2}+\left(z^{2}+x y+x z+y z\right)^{2} \geq 0$

while when $t=-3 \Rightarrow c(-3)=-8, \quad b(-3)=3=b(-1), \quad p=\frac{7}{5}, \quad q=-\frac{3}{5} \quad$ and as $k=-1$ from (7)

$w_{4}+2 w_{3}+3 w_{2}-8 w_{1}=\frac{1}{2}\left[(X-Y)^{2}+(Y-Z)^{2}+(Z-X)^{2}\right]=$

$=\frac{1}{2}\left[(x-y)^{2}(x+y+2 z)^{2}+(y-z)^{2}(y+z+2 x)^{2}+(z-x)^{2}(z+x+2 y)^{2}\right] \geq 0$.

Analogously when $a=\frac{19}{4}, \quad t=-1 \quad$ and $t=-4 \quad$ we obtain the inequalities:

$w_{4}+\left(\frac{19}{4}\right) w_{3}+\left(\frac{17}{2}\right) w_{2}+19 w_{1} \geq 0 \quad$ and $\quad w_{4}+\left(\frac{19}{4}\right) w_{3}+\left(\frac{17}{2}\right) w_{2}+\left(\frac{49}{4}\right) w_{1} \geq 0$

Remark. The parameter $t$ generates different optimal inequalities.

1. When $t=-a-1$ we have

$$
\begin{gathered}
w_{4}+a w_{3}+\left(a^{2}-1\right) w_{2}-\left(a^{2}+2 a\right) w_{1}= \\
=\frac{1}{2}\left((x-y)^{2}(x+y+a z)^{2}+(y-z)^{2}(y+z+a x)^{2}+(z-x)^{2}(z+x+a y)^{2}\right) .
\end{gathered}
$$

2. When $t=t_{1} \quad$ or $\quad t=t_{2}$ and $a \in(-\infty ;-2] \cup[4 ;+\infty) \Rightarrow b=\frac{a^{2}}{4}+2, \quad c=\frac{a^{2}}{2}+a$, $k=2, \quad 2 p+q=\frac{a}{2}$ and according to (17) we have

$$
\begin{gathered}
w_{4}+a w_{3}+\left(\frac{a^{2}}{4}+2\right) w_{2}-\left(\frac{a^{2}}{2}+a\right) w_{1}= \\
=(X+Y+Z)^{2}=\left(x^{2}+y^{2}+z^{2}+\frac{a}{2}(x y+y z+z x)\right)^{2} .
\end{gathered}
$$

3. When $t=-2$ and $a \in[1 ; 4]$ we have

$$
\begin{gathered}
w_{4}+a w_{3}+2(a-1) w_{2}+(5 a-8) w_{1}= \\
=(x+y+z)^{2}\left(x^{2}+y^{2}+z^{2}+(a-2)(x y+y z+z x)\right) .
\end{gathered}
$$

4. When $t=-1$ and $a \in[0 ; 6] \Rightarrow p=q=1, k=-1+\frac{a}{2}$ and we have

$$
w_{4}+a w_{3}+(2 a-1) w_{2}+4 a w_{1} \geq 0
$$

5. When $t=0$ and $a \in[-4 ;-1] \Rightarrow p=-1, q=0, k=-a-2$ and we have

$$
w_{4}+a w_{3}-2(a+1) w_{2}-a w_{1} \geq 0 .
$$


Section II. (nonnegative real variables)

Remark. In the proof we have in mind that the following fundamental symmetric homogeneous polinomial inequalities of degree four for three nonnegative real variables hold: $2 w_{4} \geq w_{3} \geq 2 w_{2} \geq w_{1} \geq 0$, and the inequality I. Schur [11] $w_{4}+w_{1} \geq w_{3}$.

Proof of Lemma 5. From $f(1,1,1) \geq 0 \Rightarrow c \geq-2 a-b-1$.

From $0 \leq f(x, 1,0)=x^{4}+1+a\left(x^{3}+x\right)+b x^{2} \Rightarrow x^{2}+x^{-2}+a\left(x+x^{-1}\right)+b \geq 0$.

We set $u=x+x^{-1} \geq 2$. When $a \geq-4$ and $u=2 \Rightarrow b \geq-2(a+1)$.

When $a \leq-4$ and $u=-\frac{a}{2} \geq 2 \Rightarrow b \geq \frac{a^{2}}{4}+2$.

Proof of Theorem 6. The necessity follows from Lemma 5.

Using the inequalities: $\quad w_{4}+w_{1} \geq w_{3}, w_{3} \geq 2 w_{2}$ and $w_{2} \geq w_{1} \geq 0$ we see that the conditions $2 a+2+b \geq 0$ and $1+2 a+b+c \geq 0$ are also sufficient:

$$
\begin{gathered}
f=w_{4}+a w_{3}+b w_{2}+c w_{1}=\left(w_{4}+w_{1}-w_{3}\right)+ \\
+(a+1)\left(w_{3}-2 w_{2}\right)+(2 a+2+b)\left(w_{2}-w_{1}\right)+(2 a+b+c+1) w_{1} \geq 0 .
\end{gathered}
$$

Proof of Theorem 7. Necessity.

$f(x, y, z)=w_{4}+a w_{3}+b(t) w_{2}+c w_{1}=w_{4}+a w_{3}+b(t) w_{2}+c(t) w_{1}+(c-c(t)) w_{1}$.

From $0 \leq f(t, 1,1)=0+(c-c(t)) t(t+2)$ when $t>0$ follows that $c \geq c(t)$

When $t=0$ then $c(0)=-a, \quad b(0)=-2(a+1), \quad a \in(-4 ;-1)$.

From $0 \leq \lim _{x \rightarrow+0} \frac{f(x, 1,1)}{x}=2(c+a)$ it follows that $c \geq-a=c(0)$.

Sufficiency follows analogously as in Theorem 3 from the indentity:

$$
w_{4}+a w_{3}+b(t) w_{2}+c(t) w_{1}=X^{2}+Y^{2}+Z^{2}+k(X Y+Y Z+Z X),
$$

defined with formulas (7), where $-1 \leq k \leq 2$, because each of the three cases are subregions of these of Theorem 3 :

(7.1) $-2 \leq a<-1$ and $t \in[0 ;-a-1]$ contains itself in

(3.2) that is $-2 \leq a<-\frac{1}{2}$ and $t \in\left(-\frac{1}{2} ;-a-1\right]$;

(7.2) $-4<a<-2$ and $t \in\left[0 ; t_{1}\right] \cup\left[-a-1 ; t_{2}\right]$ and $(7.3) a \leq-4$ and $t \in\left[-a-1 ; t_{2}\right]$ contain itself in $(3.4) a<-2$ and $t \in\left(-\frac{1}{2} ; t_{1}\right] \cup\left[-a-1 ; t_{2}\right]$.

\section{References}

[1] T. Ando, Some Homogeneous Cyclic Inequalities of Three Variable of Degree Three and Four, The Australian Journal of Math. Anal. and Appl. 7, (2), art. 12, (2011).

[2] T. Ando, Cubic and quartic cyclic homogeneous inequalities of Three Variables, Math. Inequal. Appl. 16, 127-142 (2013).

[3] T. Ando, Discriminants of Cyclic Homogeneous Inequalities of Three Variables, Preprint, (29 Sep. 2015) http://www.math.s.chiba-u.ac.jp/ando/ineq17.pdf

[4] V. Cirtoaje, On the Cyclic Homogeneous Polynomial Inequalities of Degree Four, Journal of Inequalities in Pure and Applied Mathematics 10 (3), art. 67, (2009). 
[5] V. Cirtoaje, Necessary and sufficient conditions for symmetric homogeneous polynomial inequalities of degree four and six in real variables, J. Nonlinear Sci. Appl., 5, 307-320 (2012).

[6] V. Cirtoaje, Necessary and Sufficient Conditions for Symmetric Homogeneous Polynomial Inequalities in Nonnegative Real Variables, Math. Inequal. Appl., 16, 413-438 (2013).

[7] V. Cirtoaje, A Strong Method for Symmetric Homogeneous Polynomial Inequalities of Degree Six in Nonnegative Real Variables, Brit. J. Math. \& Comp. Sci., 4, 685703 (2014).

[8] V. Cirtoaje, Y. Zhou, Necessary and sufficient conditions for cyclic homogeneous polynomial inequalities of degree four in real variables, The Australian Journal of Mathematical Analysis and Applications, 9 (1) Article 15, pp. 1-17, (2012).

[9] M. Milev, N. Milev, On the Cyclic Homogeneous Polynomial Inequalities of Degree Four of Three Nonnegative Real Variables, 2015, accepted in Journal of Mathematical Inequalities, http://jmi.ele-math.com/.

[10] Y. Zhou, V. Cirtoaje, Some strong sufficient conditions for cyclic homogeneous polynomial inequalities of degree four in nonnegative variables, J. Nonlinear Sci. Appl., 6 (2013).

[11] I. Schur: http://en.wikipedia.org/wiki/Schur\%27s_inequality. 